\title{
Comparison between the performances of daylight linked control system at two different latitudes \\ Marco Beccali ${ }^{1}$, Marina Bonomolo ${ }^{2}$, Giuliana Leone ${ }^{2}$ and Gaetano Zizzo ${ }^{2}$ \\ 1 D'ARCH-University of Palermo, Palermo (Italy) \\ 2 Department of Engineering-University of Palermo, Palermo (Italy)
}

\begin{abstract}
The aim of this work is to compare the performance of a daylight-linked control system installed and tested in two different case studies by means of a set of indices. The two case studies are characterized by different geometry, location and windows orientation. The first one is located at the ENEA premises in Lampedusa (IT, $35^{\circ} 30^{\prime} \mathrm{N}$ ); the second one is located in Palermo (IT, $38^{\circ} 6^{\prime} \mathrm{N}$ ) on the third floor of the building 9 of the Department of Engineering if the University of Palermo. In both cases, the indices were calculated by using the same daylight-linked control system and the same end-use (office). The results were reported and analysed in order to demonstrate as the performances of the control system can be different from the ideal performance and that they can be different according to the installation. Furthermore, according to the first outcomes, it results that a detailed analysis of the space before the installation of the control system is a necessary practice.
\end{abstract}

Keywords: lighting, daylight-linked control system-daylight- building automation control

\section{Introduction}

Building automation control systems can be used to control HVAC systems, lighting systems, video surveillance and other systems of the buildings.

The installation of these systems can be a great action to increase energy savings in buildings, to monitor the plants and to improve occupants' satisfaction (Domingues et al., 20 16). Nevertheless, the performance of these systems can decrease because of many factors (Bellia \& Fragliasso, 2015). Jain and Garg (Jain and Garg, 2018) analysed the performance and feasibility of various daylight prediction methods and their application in controlling blinds and integrated lighting system. They focused on simulation assisted open-loop control techniques that employ real-time daylight estimation methods.

The daylight-linked control systems are able to control the luminous flux based on the daylight contribution. Recent studies (Bonomolo, 2017) (Bellia, 2017) demonstrated that sensors location could influence the actual performance of the control systems. Indeed, these systems are configurated in order to achieve a certain illuminance value on the task-zone (e.g. on the workplane). For obvious reasons, the sensor cannot be placed in that position. Usually, they are placed on the ceiling or on the wall where the illuminance values are far different from the illuminance values on the task area. This fact is often the principal cause of the difference between the ideal performance and the actual ones.

Indeed, very often, the illuminance on work-plane is not fully correlated with illuminance measured by the photosensor used to control the luminaires. This fact leads to wrong information for the daylight linked control systems (DLC) affecting its efficacy. Beccali et al. (Beccali et al., 2018) applied the artificial intelligence of Neural Networks to provide a method for finding good relationships between the illuminance on workplane and the one measured in another surface.

Moreover, authors have developed a set of indices able to evaluate the actual performances of such systems in terms of luminous control efficacy and energy efficiency (Bonomolo et al., 2017). This paper presents the application of this method in two case studies with the aim of comparing the results related to a given DLC system in different contexts. To do this, the absorbed power and the illuminance values on the workplane and on the ceiling (where the photosensors were installed) were measured for two weeks long period for both case studies. 


\section{Case studies description}

The first case study (A) is concerning an office space. It is located in the lighthouse of Lampedusa (IT, $35^{\circ} 30^{\prime}$ $\mathrm{N}$ ) and has an area of $20 \mathrm{~m} 2$. It has a window located in the north facade.

Previously, four pendant luminaires were installed equipped by fluorescent sources. Each lamp was characterized by an absorbed power of $170 \mathrm{~W}$ (for a total of $680 \mathrm{~W}$ ), a luminous flux of $6550 \mathrm{~lm}$ and a colour temperature of $4000 \mathrm{~K}$. The graph in figure 1 shows a daily absorbed power variation. Except for an initial peak, it is possible to see that the absorbed power is constant for all day.

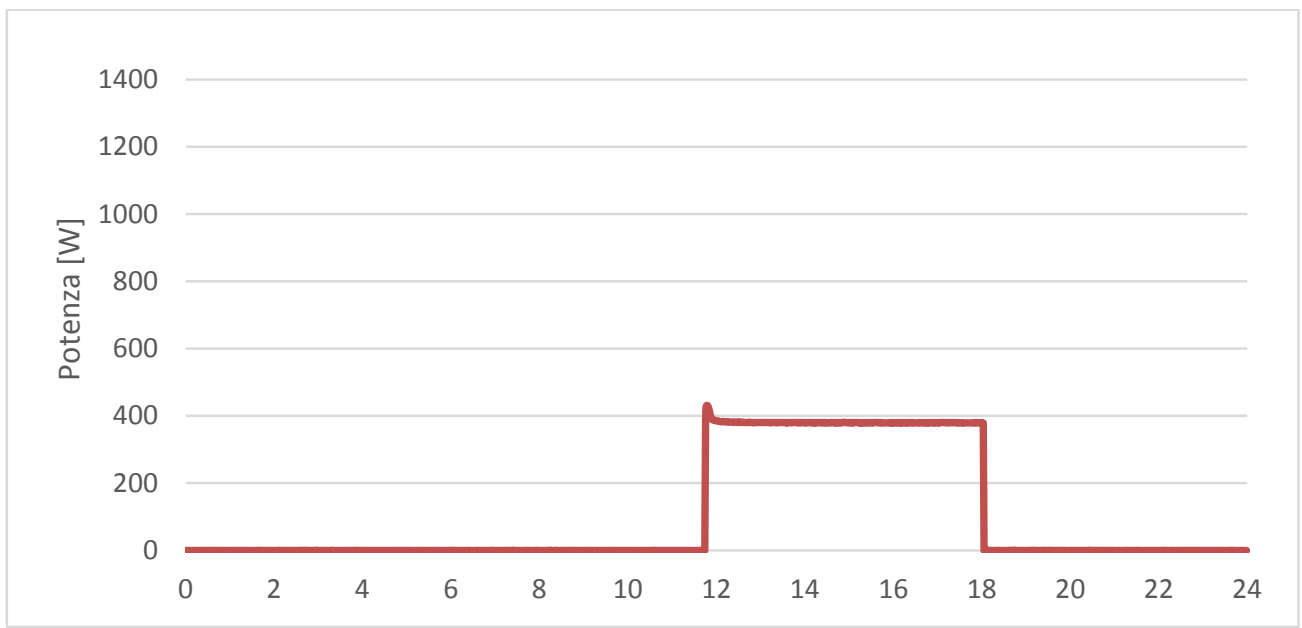

Fig. 1 Daily power absorbed by the luminaires.

They were replaced with more efficient LED luminaires. Each luminaire is characterized by an absorbed power of $33 \mathrm{~W}$ and of a luminous flux of $3960 \mathrm{~lm}$.

The second case study is the Solarlab laboratory. It is located on the third floor of the building hosting the DEIM of the University of Palermo, Italy $\left(38^{\circ} 6^{\prime} \mathrm{N}\right)$. The area is $106 \mathrm{~m} 2$ and the height is $4.40 \mathrm{~m}$ including the false ceiling, and $3.40 \mathrm{~m}$ excluding the false ceiling. Across the long side, four windows $(2.40 \times 2.90 \mathrm{~m})$ are present. In the laboratory, the following lighting luminaires are installed: four suspended luminaires equipped by LED (each one with a power of 54W) and four mono optic indoor LED spotlights $(15 \mathrm{~W})$. The first luminaries are equipped with micro-lens optics in a polycarbonate cover. The initial luminous flux declared by the manufacturer is $3600 \mathrm{~lm}$. Both luminaires have a colour temperature of $3000 \mathrm{~K}$ and a colour rendering index of $\geq 80$. The luminaires have been selected in order to achieve for each zone the illuminance values, suggested by the EN 12464 (EN 12464, 2011) standard, for the office case. Preliminary calculations have been performed using the lighting simulation software DIALUX in order to design the lighting system. For the control, a DIMLITE control system (produced by Zumtobel group) was installed. The system was composed by an electronic ballast and a open-loop photosensor. Two Delta Ohm HD2021T were installed on the workplane and close to the photosensor. Figure 2 shows some pictures of the cases study, the plans with the position of the luminaires and sensors. The photosensors are controlled by an open loop algorithm and are installed "look out". Even though the photosensors are calibrated, they may not work ideally. By the analysis of the illuminance values measured during a cloudy sky day on the ceiling and the task area, it was possible to observe that with only artificial light or with diffuse daylight, a certain correlation can be derived by data. On the contrary, analysing the illuminance values measured during a clear sky day on the ceiling and the task area, it is worth noting that very high values of illuminance on the task area make the relationship with illuminance on the ceiling no longer reliable. Therefore, mainly during clear sky day, the actual performance of the DLCs can be very different from the ideal ones.

During the experimental campaign, the following data have measured: indoor illuminances, apparent, active and reactive power of the lighting circuit as well as electric current. Data have been collected through the platform LabVIEW System Design.

Two Delta Ohm HD $2021 \mathrm{~T}$ (measuring range 0.02-20 klx) have been installed in each case study to monitor the illuminance values during the experimental campaign. Electrical consumption has been measured using 
SIEMENS SENTRON Power Monitoring Device PAC3200, an instrument for measuring electric power distribution variables such as voltage, current, power, electrical work and frequency.

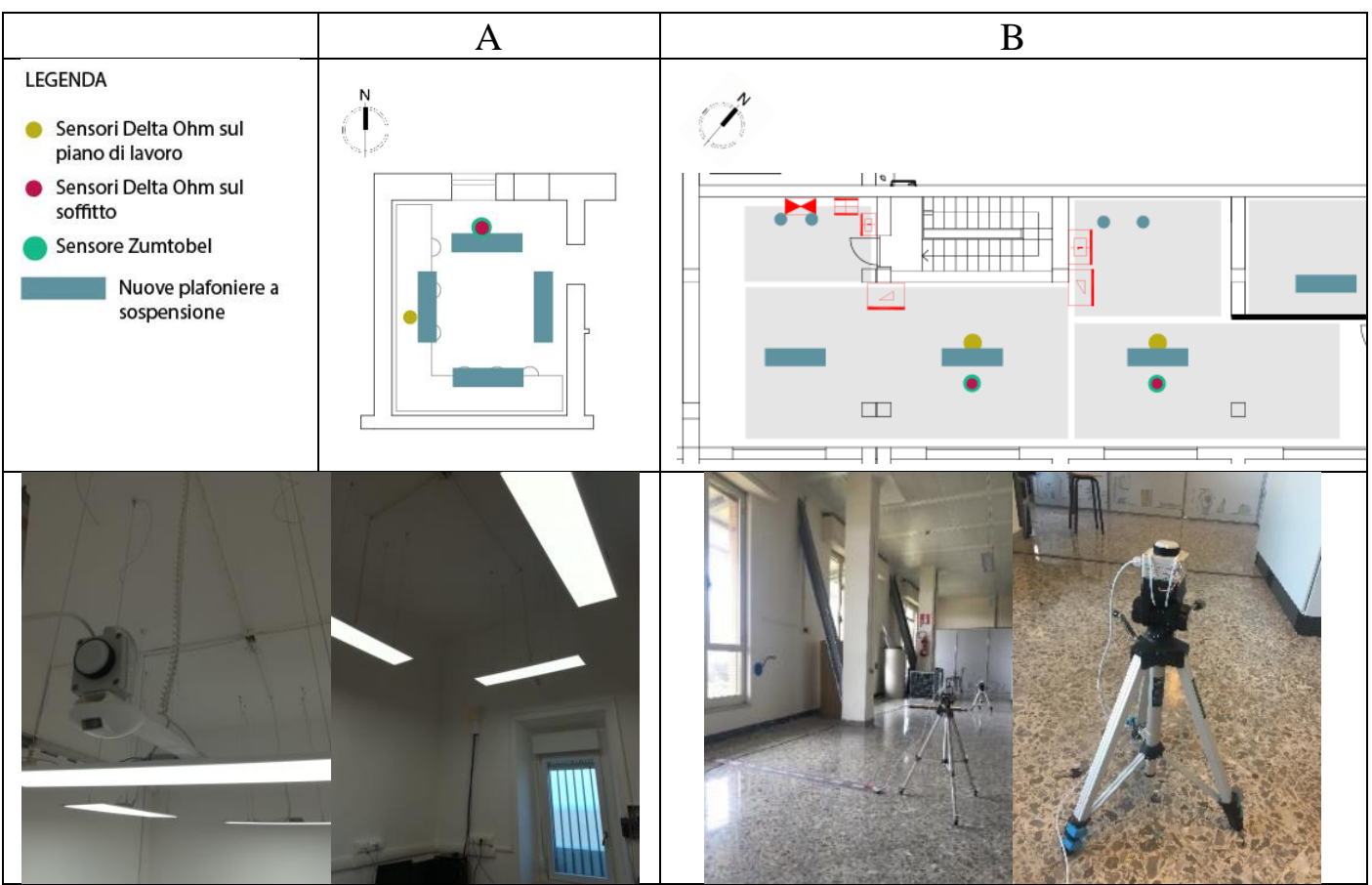

Fig. 2: Plans of case studies with location of luminaires and sensors (above) and pictures of case studies (below).

\section{Indices}

The set of indices used to calculate the performance of the system has been presented in previous work (Bonomolo, 2017) and here summarized. The set of indices includes four elements. The first one is called ALD (Artificial Light Demand). It can be defined as the sum, during the operation time, of the differences between the illuminance target value on task area and illuminance due to available natural light, when this one is lower than the set point itself. The ratio between the actual electricity consumption and ALD $(\mathrm{lx} \cdot \mathrm{h})$ defines the second index called ERI (Energy Ratio of Illuminance). This index can be calculated to know how the system worked respect it should have ideally work. The actual performances of the system are lower than the ideal ones because it could be a problem of over-illuminance and under-illuminance. For these reasons, authors define the other two indices. The first one, named OAR (Over illuminance Avoidance Ratio), is defined as the ratio, evaluated for an observed time, between the minimum requirement of artificial light (ALD) and the sum of it plus the artificial light eventually provided in excess (Eexc). This index gives an idea all the times the illuminance of the artificial light overcomes the task illuminance, causing a "over-lighting". A second index can be considered for accounting the relative weight of the "under-lighting" times. It occurs when Etot $<$ Eset and Enat $<$ Eset and the system are not capable of fulfilling the minimum target illuminance. This second index is called UAR (Under-illuminance Avoidance Ratio).

\section{Results and discussion}

The indices were calculated implementing the dimmer and on-off control strategies. In the first case, the values measured were used to calculate the indices. In the second case, to calculate the indices with daylight control, but without the luminous flux regulation, it was supposed that the luminaires were at $100 \%$ of power when they were turned on according to the daylight contribution. The indices were calculated considering all day and considering only the afternoon time. In figure 3, 4, 5, and 6 the illuminance values measured for different days and strategies. In particular, the 13th September was selected as days with high daylight contribution and, therefore, a low value of ALD. While, the 26th September was selected as days with low daylight contribution and, therefore, a high value of ALD. 


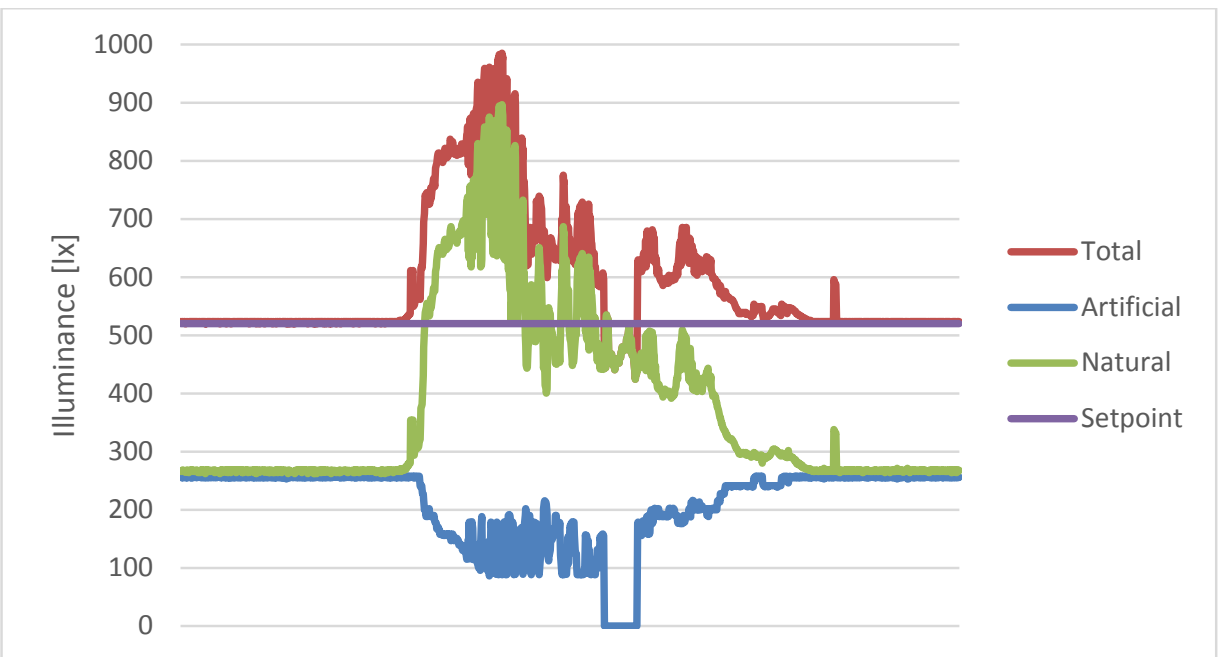

Fig. 3: Illuminance values measured on 13th September with dimmer control strategy in case study A.

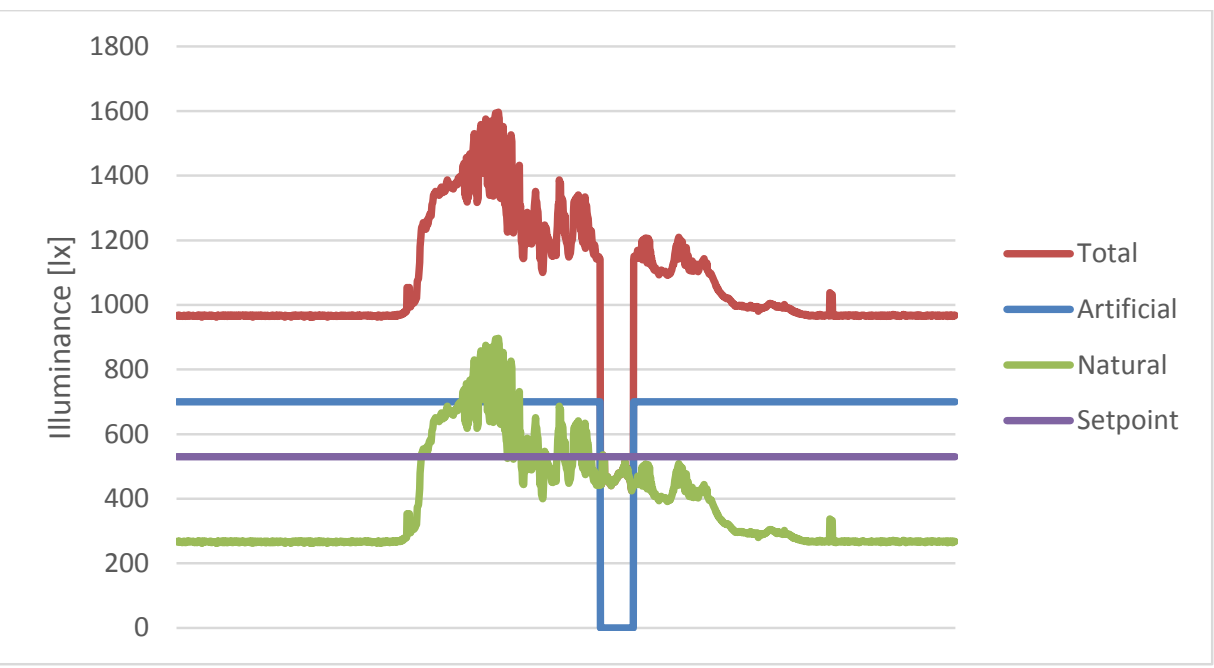

Fig. 4: Illuminance values measured on 13th September with on-off control strategy in case study A.

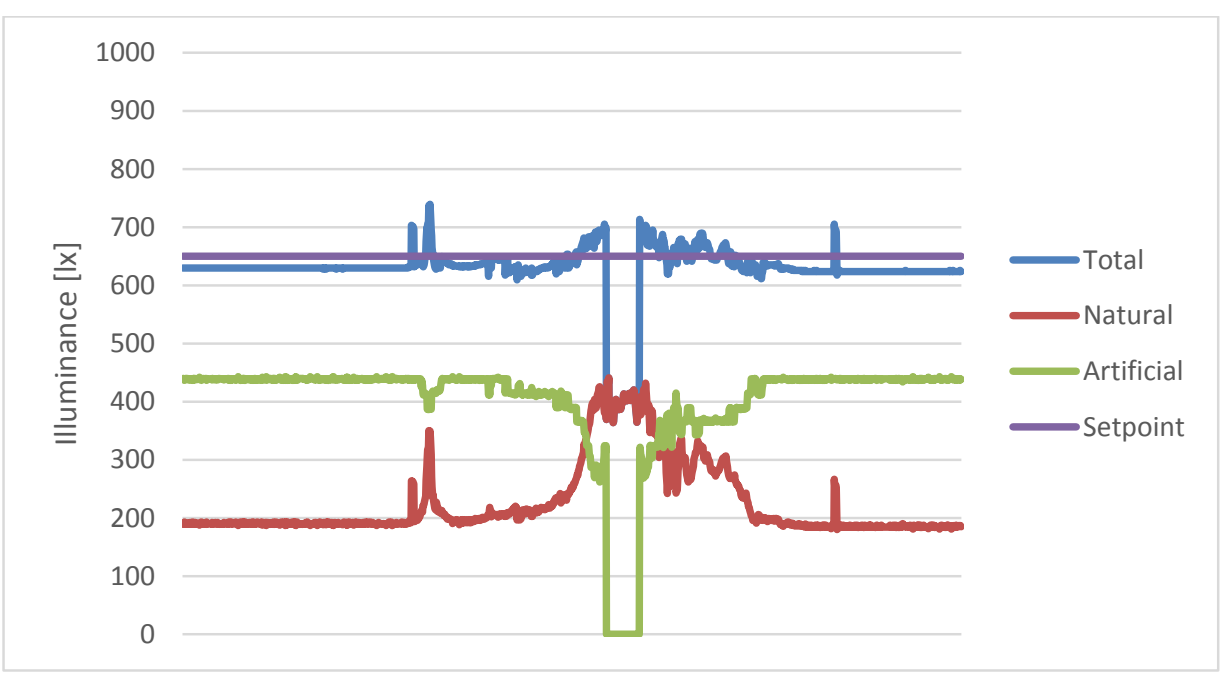

Fig. 5: Illuminance values measured on 26th September with on-off control strategy in case study A. 


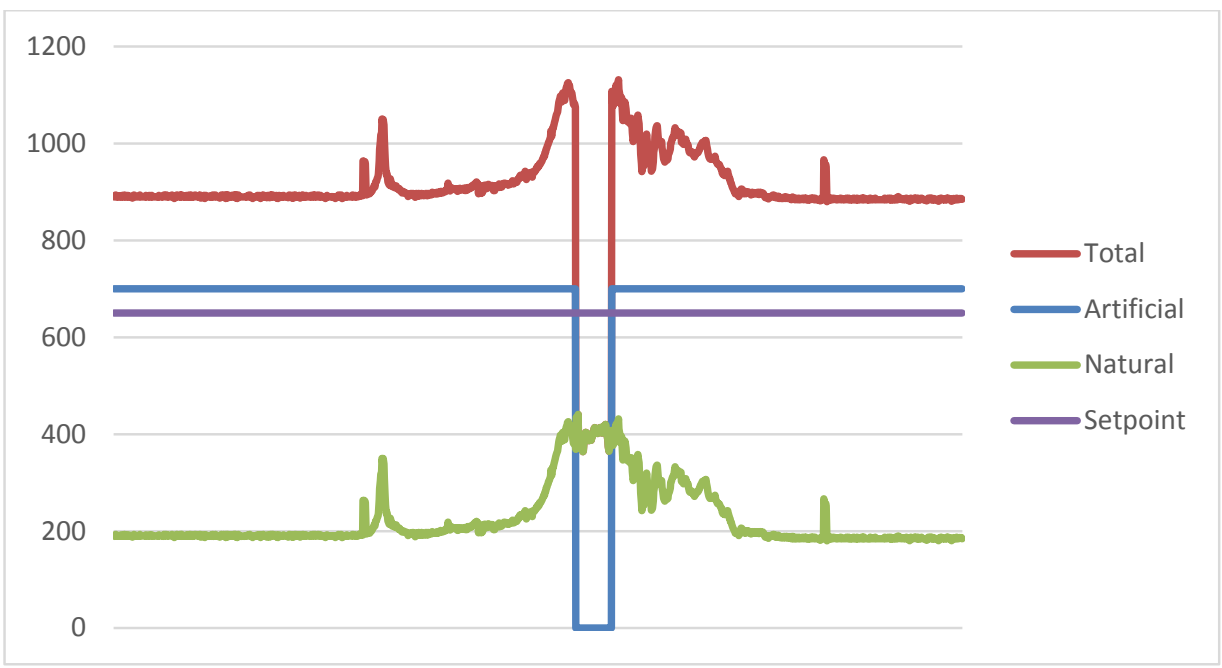

Fig. 6: Illuminance values measured on 26th September with on-off control strategy in case study A.

In general, these indices can be useful to understand the actual performances of the control systems in terms of consumption and visual comfort. In particular, the comparison between the indices calculated for the two cases study highlights that the same control system can have different performances if installed with different conditions (e.g. room sizes, latitude and orientation).

The indices were calculated for typical office schedules ( 8 hours and 1 hour of lunch break). From a first analysis, it is possible to note that the system worked reasonably well in both cases, but, as predicted, not ideally. In table 1 the indices calculated for the case study in Lampedusa and Table 2 the indices calculated for the case study of Palermo are shown.

It is possible to note that, in general, high values of OAR correspond to small values of ERI. Looking at the valued calculated for case study A, it is possible to see that the OAR values range from 0.33 to 0.94 for the dimmer strategy. The comparison of the values plotted in graphs in figure 7 shows that the system works better in Palermo in terms of over-illuminance even if there is a more precise relation between ERI and OAR calculated for the case of Lampedusa. The performance in terms of OAR were deficient in case of on-off strategy in Lampedusa. Indeed, the OAR ranged from 0.09 to 0.51 . On the contrary, the system operating in on-off mode, worked better in the case study in Palermo with good values of OAR.

Tab. 1: Indices calculated for the case study in Lampedusa.

\begin{tabular}{|c|c|c|c|c|c|c|c|c|c|c|c|}
\hline & & & \multicolumn{3}{|c|}{ Dimmer control } & \multicolumn{3}{|c|}{ On-off control } \\
\hline Schedules & Date & ALD & OAR & UAR & $\begin{array}{c}\text { ELEC } \\
{[\mathrm{Wh}]}\end{array}$ & $\begin{array}{c}\text { ERI } \\
{[\mathrm{Wh} / \mathrm{l} \cdot \mathrm{h}]}\end{array}$ & OAR & UAR & $\begin{array}{c}\text { ELEC } \\
{[\mathrm{Wh}]}\end{array}$ & $\begin{array}{c}\text { ERI } \\
{[\mathrm{Wh} / \mathrm{l} \cdot \mathrm{h}]}\end{array}$ \\
\hline $\begin{array}{l}09: 00-13: 00 \\
14: 00-17: 00\end{array}$ & $\begin{array}{c}10 / 09 / 18 \\
\text { (Full day) }\end{array}$ & 2723 & 0.80 & 0.81 & 605 & 0.22 & 0.14 & 1 & 1003 & 1.37 \\
\hline $\begin{array}{c}09: 00-13: 00 \\
14: 00-17: 00\end{array}$ & $\begin{array}{c}10 / 09 / 18 \\
\text { (Afternoon) }\end{array}$ & 1366 & 0.75 & 1 & 233 & 0.27 & 0.24 & 1 & 383 & 0.82 \\
\hline $\begin{array}{c}09: 00-13: 00 \\
14: 00-17: 00\end{array}$ & $\begin{array}{c}11 / 09 / 18 \\
\text { (Full day) }\end{array}$ & 1389 & 0.72 & 0.73 & 356 & 0.26 & 0.24 & 1 & 1003 & 0.80 \\
\hline $\begin{array}{l}09: 00-13: 00 \\
14: 00-17: 00\end{array}$ & $\begin{array}{c}11 / 09 / 18 \\
\text { (Afternoon) }\end{array}$ & 582 & 0.67 & 1 & 233 & 0.33 & 0.27 & 1 & 383 & 0.73 \\
\hline $\begin{array}{l}09: 00-13: 00 \\
14: 00-17: 00\end{array}$ & $\begin{array}{c}12 / 09 / 18 \\
\text { (Full day) }\end{array}$ & 503 & 0.43 & 1 & 373 & 0.74 & 0.09 & 1 & 585 & 1.16 \\
\hline $\begin{array}{l}09: 00-13: 00 \\
14: 00-17: 00\end{array}$ & $\begin{array}{c}12 / 09 / 18 \\
\text { (Afternoon) }\end{array}$ & 364 & 0.55 & 1 & 136 & 0.54 & 0.17 & 1 & 224 & 0.61 \\
\hline $\begin{array}{c}09: 00-13: 00 \\
14: 00-17: 00\end{array}$ & $\begin{array}{c}13 / 09 / 18 \\
\text { (Full day) }\end{array}$ & 433 & 0.33 & 1 & 406 & 0.94 & 0.09 & 1 & 585 & 1.20 \\
\hline $\begin{array}{l}09: 00-13: 00 \\
14: 00-17: 00\end{array}$ & $\begin{array}{c}13 / 09 / 18 \\
\text { (Afternoon) }\end{array}$ & 317 & 0.53 & 1 & 136 & 0.58 & 0.17 & 1 & 224 & 0.65 \\
\hline
\end{tabular}




\begin{tabular}{|c|c|c|c|c|c|c|c|c|c|c|}
\hline $\begin{array}{l}09: 00-13: 00 \\
\text { 14:00-17:00 }\end{array}$ & $\begin{array}{c}14 / 09 / 18 \\
\text { (Full day) }\end{array}$ & 682 & 0.40 & 0.99 & 505 & 0.74 & 0.12 & 1 & 590 & 0.86 \\
\hline $\begin{array}{l}09: 00-13: 00 \\
14: 00-17: 00\end{array}$ & $\begin{array}{c}14 / 09 / 18 \\
\text { (Afternoon) }\end{array}$ & 289 & 0.34 & 0.97 & 136 & 0.85 & 0.14 & 1 & 225 & 0.78 \\
\hline $\begin{array}{l}09: 00-13: 00 \\
\text { 14:00-17:00 }\end{array}$ & $\begin{array}{c}16 / 09 / 18 \\
\text { (Full day) }\end{array}$ & 931 & 0.59 & 0.99 & 471 & 0.51 & 0.17 & 1 & 762 & 0.82 \\
\hline $\begin{array}{l}09: 00-13: 00 \\
14: 00-17: 00\end{array}$ & $\begin{array}{c}16 / 09 / 18 \\
\text { (Afternoon) }\end{array}$ & 642 & 0.75 & 1 & 177 & 0.38 & 0.31 & 1 & 291 & 0.45 \\
\hline $\begin{array}{l}09: 00-13: 00 \\
14: 00-17: 00\end{array}$ & $\begin{array}{c}17 / 09 / 18 \\
\text { (Full day) }\end{array}$ & 1391 & 0.66 & 0.99 & 467 & 0.34 & 0.25 & 1 & 762 & 0.55 \\
\hline $\begin{array}{l}09: 00-13: 00 \\
14: 00-17: 00\end{array}$ & $\begin{array}{c}17 / 09 / 18 \\
\text { (Afternoon) }\end{array}$ & 965 & 0.86 & 1 & 177 & 0.25 & 0.46 & 1 & 291 & 0.30 \\
\hline $\begin{array}{l}09: 00-13: 00 \\
14: 00-17: 00\end{array}$ & $\begin{array}{c}18 / 09 / 18 \\
\text { (Full day) }\end{array}$ & 1297 & 0.62 & 0.99 & 463 & 0.36 & 0.23 & 1 & 762 & 0.59 \\
\hline $\begin{array}{l}09: 00-13: 00 \\
14: 00-17: 00\end{array}$ & $\begin{array}{c}18 / 09 / 18 \\
\text { (Afternoon) }\end{array}$ & 893 & 0.83 & 1 & 177 & 0.26 & 0.43 & 1 & 291 & 0.33 \\
\hline $\begin{array}{l}09: 00-13: 00 \\
\text { 14:00-17:00 }\end{array}$ & $\begin{array}{c}19 / 09 / 18 \\
\text { (Full day) }\end{array}$ & 1376 & 0.64 & 0.99 & 479 & 0.35 & 0.25 & 1 & 767 & 0.56 \\
\hline $\begin{array}{l}09: 00-13: 00 \\
14: 00-17: 00\end{array}$ & $\begin{array}{c}19 / 09 / 18 \\
\text { (Afternoon) }\end{array}$ & 946 & 0.83 & 1 & 177 & 0.26 & 0.45 & 1 & 293 & 0.31 \\
\hline $\begin{array}{l}09: 00-13: 00 \\
\text { 14:00-17:00 }\end{array}$ & $\begin{array}{c}20 / 09 / 18 \\
\text { (Full day) } \\
\end{array}$ & 1745 & 0.71 & 1 & 537 & 0.31 & 0.31 & 1 & 762 & 0.44 \\
\hline $\begin{array}{l}09: 00-13: 00 \\
\text { 14:00-17:00 }\end{array}$ & $\begin{array}{c}20 / 09 / 18 \\
\text { (Afternoon) }\end{array}$ & 995 & 0.90 & 1 & 177 & 0.24 & 0.47 & 1 & 291 & 0.29 \\
\hline $\begin{array}{l}09: 00-13: 00 \\
\text { 14:00-17:00 }\end{array}$ & $\begin{array}{c}21 / 09 / 18 \\
\text { (Full day) }\end{array}$ & 1890 & 0.71 & 1 & 571 & 0.30 & 0.34 & 1 & 762 & 0.40 \\
\hline $\begin{array}{l}09: 00-13: 00 \\
14: 00-17: 00\end{array}$ & $\begin{array}{c}21 / 09 / 18 \\
\text { (Afternoon) }\end{array}$ & 951 & 0.81 & 1 & 177 & 0.26 & 0.45 & 1 & 291 & 0.31 \\
\hline $\begin{array}{l}09: 00-13: 00 \\
\text { 14:00-17:00 }\end{array}$ & $\begin{array}{c}22 / 09 / 18 \\
\text { (Full day) }\end{array}$ & 1950 & 0.76 & 1 & 555 & 0.28 & 0.35 & 1 & 762 & 0.39 \\
\hline $\begin{array}{l}09: 00-13: 00 \\
14: 00-17: 00\end{array}$ & $\begin{array}{c}22 / 09 / 18 \\
\text { (Afternoon) }\end{array}$ & 1002 & 0.86 & 1 & 177 & 0.25 & 0.48 & 1 & 291 & 0.29 \\
\hline $\begin{array}{l}09: 00-13: 00 \\
14: 00-17: 00\end{array}$ & $\begin{array}{c}23 / 09 / 18 \\
\text { (Full day) }\end{array}$ & 1382 & 0.66 & 0.99 & 466 & 0.34 & 0.25 & 1 & 767 & 0.55 \\
\hline $\begin{array}{l}09: 00-13: 00 \\
\text { 14:00-17:00 }\end{array}$ & $\begin{array}{c}23 / 09 / 18 \\
\text { (Afternoon) }\end{array}$ & 934 & 0.85 & 1 & 177 & 0.25 & 0.44 & 1 & 293 & 0.31 \\
\hline $\begin{array}{l}09: 00-13: 00 \\
14: 00-17: 00\end{array}$ & $\begin{array}{c}24 / 09 / 18 \\
\text { (Full day) }\end{array}$ & 1738 & 0.59 & 1 & 631 & 0.36 & 0.31 & 1 & 762 & 0.44 \\
\hline $\begin{array}{l}09: 00-13: 00 \\
14: 00-17: 00\end{array}$ & $\begin{array}{c}24 / 09 / 18 \\
\text { (Afternoon) }\end{array}$ & 992 & 0.64 & 1 & 177 & 0.32 & 0.47 & 1 & 291 & 0.29 \\
\hline $\begin{array}{l}09: 00-13: 00 \\
\text { 14:00-17:00 }\end{array}$ & $\begin{array}{c}25 / 09 / 18 \\
\text { (Full day) }\end{array}$ & 1351 & 0.69 & 0.96 & 437 & 0.32 & 0.24 & 1 & 939 & 0.70 \\
\hline $\begin{array}{l}09: 00-13: 00 \\
\text { 14:00-17:00 }\end{array}$ & $\begin{array}{c}25 / 09 / 18 \\
\text { (Afternoon) }\end{array}$ & 825 & 0.81 & 1 & 168 & 0.27 & 0.39 & 1 & 359 & 0.44 \\
\hline $\begin{array}{l}09: 00-13: 00 \\
\text { 14:00-17:00 }\end{array}$ & $\begin{array}{c}26 / 09 / 18 \\
\text { (Full day) }\end{array}$ & 2805 & 0.94 & 0.98 & 619 & 0.22 & 0.51 & 1 & 726 & 0.25 \\
\hline $\begin{array}{l}09: 00-13: 00 \\
14: 00-17: 00\end{array}$ & $\begin{array}{c}26 / 09 / 18 \\
\text { (Afternoon) }\end{array}$ & 1022 & 0.96 & 0.99 & 168 & 0.23 & 0.49 & 1 & 277 & 0.27 \\
\hline
\end{tabular}


Tab. 2: Indices calculated for the case study in Palermo.

\begin{tabular}{|c|c|c|c|c|c|c|c|c|c|c|}
\hline $\begin{array}{c}\text { Scenario } \\
\text { time }\end{array}$ & Scenario data & $\begin{array}{l}\text { ALD } \\
{[1 \mathrm{x} \cdot \mathrm{h}]}\end{array}$ & OAR & UAR & $\begin{array}{l}\text { ELEC } \\
\text { [Wh] }\end{array}$ & $\begin{array}{c}\text { ERI } \\
{[\mathrm{Wh} / \mathrm{l} \mathrm{x} \cdot \mathrm{h}]}\end{array}$ & OAR & UAR & $\begin{array}{c}\text { ELEC } \\
{[\mathrm{Wh}]}\end{array}$ & $\begin{array}{c}\text { ERI } \\
{[\mathrm{Wh} / \mathrm{lx} \cdot \mathrm{h}]}\end{array}$ \\
\hline \multirow[t]{2}{*}{$\begin{array}{l}09: 00-13: 00 \\
15: 00-18: 00\end{array}$} & $\begin{array}{l}\text { 31/05/2017 } \\
\text { (Full Day) }\end{array}$ & 568.6 & 0.65 & 0.70 & 222.8 & 0.39 & 0.16 & 0.80 & 817.4 & 1.437 \\
\hline & $\begin{array}{l}31 / 05 / 2017 \\
\text { (Afternoon) }\end{array}$ & 493.6 & 0.65 & 0.65 & 163.0 & 0.33 & 0.26 & 0.91 & 421.6 & 0.85 \\
\hline \multirow[t]{2}{*}{$\begin{array}{l}09: 00-13: 00 \\
15: 00-18: 00 \\
\end{array}$} & $\begin{array}{c}01 / 06 / 2017 \\
\text { (Full Day) }\end{array}$ & 733.5 & 0.87 & 0.68 & 207.5 & 0.28 & 0.51 & 0.65 & 280.7 & 0.383 \\
\hline & $\begin{array}{l}01 / 06 / 2017 \\
\text { (Afternoon) }\end{array}$ & 675.2 & 0.86 & 0.73 & 140.0 & 0.21 & 0.49 & 0.70 & 270.7 & 0.40 \\
\hline \multirow[t]{2}{*}{$\begin{array}{l}\text { 09:00-13:00 } \\
\text { 15:00-18:00 } \\
\end{array}$} & $\begin{array}{c}02 / 06 / 2017 \\
\text { (Full Day) }\end{array}$ & 725.9 & 0.84 & 0.87 & 245.3 & 0.34 & 0.33 & 0.99 & 514.8 & 0.709 \\
\hline & $\begin{array}{l}02 / 06 / 2017 \\
\text { (Afternoon) }\end{array}$ & 703.9 & 0.90 & 0.87 & 168.8 & 0.24 & 0.39 & 0.99 & 407.1 & 0.58 \\
\hline \multirow[t]{2}{*}{$\begin{array}{l}11: 00-15: 00 \\
16: 00-20: 00 \\
\end{array}$} & $\begin{array}{c}03 / 06 / 2017 \\
\text { (Full Day) }\end{array}$ & 1613.1 & 0.73 & 0.82 & 465.2 & 0.29 & 0.39 & 0.95 & 780.9 & 0.484 \\
\hline & $\begin{array}{l}\text { 03/06/2017 } \\
\text { (Afternoon) }\end{array}$ & 1209.8 & 0.68 & 1.00 & 405.2 & 0.33 & 0.58 & 1.00 & 473.7 & 0.39 \\
\hline \multirow[t]{2}{*}{$\begin{array}{l}\text { 08:00-12:00 } \\
\text { 13:00-17:00 } \\
\end{array}$} & $\begin{array}{c}04 / 06 / 2017 \\
\text { (Full Day) }\end{array}$ & 854.2 & 0.89 & 0.42 & 127.3 & 0.21 & 0.67 & 0.40 & 792.7 & 0.215 \\
\hline & $\begin{array}{l}04 / 06 / 2017 \\
\text { (Afternoon) }\end{array}$ & 748.5 & 0.87 & 0.44 & 127.2 & 0.17 & 0.64 & 0.46 & 174.0 & 0.23 \\
\hline \multirow[t]{2}{*}{$\begin{array}{l}08: 00-12: 00 \\
13: 00-17: 01 \\
\end{array}$} & $\begin{array}{c}05 / 06 / 2017 \\
\text { (Full Day) }\end{array}$ & 469.5 & 0.62 & 0.97 & 195.6 & 0.42 & 0.21 & 1.00 & 792.7 & 1.095 \\
\hline & $\begin{array}{l}05 / 06 / 2017 \\
\text { (Afternoon) }\end{array}$ & 449.3 & 0.70 & 0.97 & 151.2 & 0.34 & 0.28 & 1.00 & 367.3 & 0.82 \\
\hline \multirow[t]{2}{*}{$\begin{array}{l}10: 30-13: 30 \\
14: 30-18: 00 \\
\end{array}$} & $\begin{array}{c}06 / 06 / 2017 \\
\text { (Full Day) }\end{array}$ & 1468.1 & 0.90 & 0.76 & 348.0 & 0.24 & 0.48 & 0.85 & 677.0 & 0.461 \\
\hline & $\begin{array}{l}06 / 06 / 2017 \\
\text { (Afternoon) }\end{array}$ & 1007.5 & 0.86 & 0.96 & 273.3 & 0.27 & 0.48 & 1.00 & 473.7 & 0.47 \\
\hline \multirow[t]{2}{*}{$\begin{array}{l}07: 00-11: 00 \\
12: 00-16: 00 \\
\end{array}$} & $\begin{array}{c}\text { 07/06/2017 } \\
\text { (Full Day) }\end{array}$ & 852.4 & 0.77 & 0.95 & 299.0 & 0.34 & 0.33 & 0.96 & 612.6 & 0.719 \\
\hline & $\begin{array}{l}07 / 06 / 2017 \\
\text { (Afternoon) }\end{array}$ & 605.0 & 0.81 & 0.93 & 187.4 & 0.31 & 0.41 & 0.94 & 367.7 & 0.61 \\
\hline \multirow[t]{2}{*}{$\begin{array}{l}07: 00-11: 00 \\
12: 00-16: 00 \\
\end{array}$} & $\begin{array}{c}\text { 08/06/2017 } \\
\text { (Full Day) }\end{array}$ & 928.9 & 0.92 & 0.30 & 148.7 & 0.16 & 0.79 & 0.23 & 115.5 & 0.124 \\
\hline & $\begin{array}{l}08 / 06 / 2017 \\
\text { (Afternoon) }\end{array}$ & 780.8 & 0.92 & 0.30 & 66.2 & 0.08 & 0.75 & 0.27 & 106.3 & 0.14 \\
\hline \multirow[t]{2}{*}{$\begin{array}{l}07: 30-11: 30 \\
12: 30-16: 30\end{array}$} & $\begin{array}{c}\text { 09/06/2017 } \\
\text { (Full Day) }\end{array}$ & 703.1 & 0.99 & 0.23 & 109.7 & 0.16 & 0.77 & 0.18 & 87.4 & 0.124 \\
\hline & $\begin{array}{l}\text { 09/06/2017 } \\
\text { (Afternoon) }\end{array}$ & 637.8 & 0.99 & 0.21 & 61.3 & 0.10 & 0.75 & 0.20 & 87.3 & 0.14 \\
\hline \multirow[t]{2}{*}{$\begin{array}{l}07: 00-11: 00 \\
12: 00-16: 00 \\
\end{array}$} & $\begin{array}{l}\text { 10/06/2017 } \\
\text { (Full Day) }\end{array}$ & 493.1 & 1.00 & 0.20 & 106.1 & 0.22 & 0.60 & 0.28 & 116.4 & 0.236 \\
\hline & $\begin{array}{l}\text { 10/06/2017 } \\
\text { (Afternoon) }\end{array}$ & 486.3 & 1.00 & 0.19 & 56.9 & 0.12 & 0.60 & 0.29 & 116.3 & 0.24 \\
\hline \multirow[t]{2}{*}{$\begin{array}{l}\text { 07:00-11:00 } \\
\text { 12:00-16:00 } \\
\end{array}$} & $\begin{array}{l}11 / 06 / 2017 \\
\text { (Full Day) }\end{array}$ & 1430.9 & 0.71 & 0.61 & 317.5 & 0.22 & 0.58 & 0.73 & 457.3 & 0.284 \\
\hline & $\begin{array}{l}11 / 06 / 2017 \\
\text { (Afternoon) }\end{array}$ & 986.6 & 0.69 & 0.87 & 307.5 & 0.31 & 0.54 & 0.97 & 447.3 & 0.42 \\
\hline \multirow[t]{2}{*}{$\begin{array}{l}10: 00-14: 00 \\
\text { 15:00-19:00 }\end{array}$} & $\begin{array}{l}15 / 06 / 2017 \\
\text { (Full Day) }\end{array}$ & 456.9 & 0.71 & 0.58 & 187.7 & 0.41 & 0.51 & 0.53 & 320.5 & 0.435 \\
\hline & $\begin{array}{l}15 / 06 / 2017 \\
\text { (Afternoon) }\end{array}$ & 426.2 & 0.70 & 0.62 & 137.7 & 0.32 & 0.47 & 0.62 & 270.5 & \\
\hline \multirow[t]{2}{*}{$\begin{array}{l}\text { 07:00-11:00 } \\
\text { 12:00-16:00 }\end{array}$} & $\begin{array}{c}\text { 05/07/2017 } \\
\text { (Full Day) }\end{array}$ & 444.6 & 1.00 & 0.10 & 142.4 & 0.22 & 0.87 & 0.10 & 126.1 & 0.182 \\
\hline & $\begin{array}{l}05 / 07 / 2017 \\
\text { (Afternoon) }\end{array}$ & 439.4 & 1.00 & 0.10 & 49.4 & 0.11 & 0.86 & 0.11 & 76.2 & 0.12 \\
\hline
\end{tabular}




\begin{tabular}{|c|c|c|c|c|c|c|c|c|c|c|}
\hline $\begin{array}{l}\text { 08:00-12:00 } \\
\text { 13:00-17:00 }\end{array}$ & $\begin{array}{c}\text { 06/07/2017 } \\
\text { (Full Day) }\end{array}$ & 444.5 & 0.55 & 1.00 & 246.1 & 0.55 & 0.26 & 1.00 & 792.7 & 0.997 \\
\hline & $\begin{array}{l}\text { 06/07/2017 } \\
\text { (Afternoon) }\end{array}$ & 444.5 & 0.57 & 1.00 & 191.2 & 0.43 & 0.28 & 1.00 & 375.7 & 0.85 \\
\hline \multirow[t]{2}{*}{$\begin{array}{l}\text { 09:00-13:00 } \\
\text { 15:00-18:00 } \\
\end{array}$} & $\begin{array}{l}\text { 13/07/2017 } \\
\text { (Full Day) }\end{array}$ & 575.8 & 0.66 & 0.58 & 227.1 & 0.39 & 0.55 & 0.54 & 338.2 & 0.388 \\
\hline & $\begin{array}{l}\text { 13/07/2017 } \\
\text { (Afternoon) }\end{array}$ & 518.1 & 0.64 & 0.64 & 177.1 & 0.34 & 0.50 & 0.65 & 288.2 & 0.40 \\
\hline \multirow[t]{2}{*}{$\begin{array}{l}\text { 07:00-11:00 } \\
\text { 12:00-16:00 }\end{array}$} & $\begin{array}{l}\text { 17/07/2017 } \\
\text { (Full Day) }\end{array}$ & 437.8 & 0.47 & 0.44 & 140.4 & 0.51 & 0.11 & 0.86 & 850.5 & 1.943 \\
\hline & $\begin{array}{l}17 / 07 / 2017 \\
\text { (Afternoon) }\end{array}$ & 423.0 & 0.78 & 0.48 & 67.2 & 0.16 & 0.23 & 0.86 & 367.7 & 0.87 \\
\hline \multirow[t]{2}{*}{$\begin{array}{l}\text { 09:00-13:00 } \\
\text { 15:00-18:00 }\end{array}$} & $\begin{array}{l}\text { 18/07/2017 } \\
\text { (Full Day) }\end{array}$ & 322.7 & 0.52 & 0.88 & 215.0 & 0.67 & 0.26 & 0.77 & 320.5 & 0.993 \\
\hline & $\begin{array}{l}18 / 07 / 2017 \\
\text { (Afternoon) }\end{array}$ & 322.7 & 0.52 & 0.88 & 165.0 & 0.51 & 0.26 & 0.77 & 270.5 & 0.84 \\
\hline \multirow[t]{2}{*}{$\begin{array}{l}\text { 09:00-13:00 } \\
\text { 15:00-18:00 }\end{array}$} & $\begin{array}{l}21 / 07 / 2017 \\
\text { (Full Day) }\end{array}$ & 644.7 & 0.70 & 0.57 & 233.4 & 0.36 & 0.16 & 0.96 & 947.7 & 1.470 \\
\hline & $\begin{array}{l}21 / 07 / 2017 \\
\text { (Afternoon) }\end{array}$ & 619.8 & 0.90 & 0.59 & 141.8 & 0.23 & 0.30 & 1.00 & 473.7 & 0.76 \\
\hline \multirow[t]{2}{*}{$\begin{array}{l}\text { 09:00-13:00 } \\
\text { 15:00-18:00 } \\
\end{array}$} & $\begin{array}{l}\text { 15/11/2017 } \\
\text { (Full Day) }\end{array}$ & 1028.0 & 0.90 & 0.72 & 284.9 & 0.28 & 0.59 & 0.77 & 417.7 & 0.406 \\
\hline & $\begin{array}{l}\text { 15/11/2017 } \\
\text { (Afternoon) }\end{array}$ & 957.27 & 0.93 & 0.72 & 214.1 & 0.22 & 0.62 & 0.77 & 332.3 & 0.35 \\
\hline \multirow[t]{2}{*}{$\begin{array}{l}\text { 09:00-13:00 } \\
\text { 15:00-18:00 } \\
\end{array}$} & $\begin{array}{c}22 / 11 / 2017 \\
\text { (Full Day) }\end{array}$ & 1743.1 & 0.84 & 0.73 & 431.5 & 0.25 & 0.41 & 0.90 & 947.7 & 0.544 \\
\hline & $\begin{array}{l}22 / 11 / 2017 \\
\text { (Afternoon) }\end{array}$ & 1534.63 & 0.92 & 0.81 & 334.9 & 0.22 & 0.74 & 1.00 & 473.7 & 0.31 \\
\hline \multirow[t]{2}{*}{$\begin{array}{l}\text { 08:00-12:00 } \\
\text { 13:00-17:00 } \\
\end{array}$} & $\begin{array}{l}23 / 11 / 2017 \\
\text { (Full Day) }\end{array}$ & 1705.8 & 0.84 & 0.76 & 435.2 & 0.26 & 0.40 & 0.91 & 947.7 & 0.556 \\
\hline & $\begin{array}{l}23 / 11 / 2017 \\
\text { (Afternoon) }\end{array}$ & 1534.17 & 0.92 & 0.84 & 339.5 & 0.22 & 0.74 & 1.00 & 473.7 & 0.31 \\
\hline \multirow[t]{2}{*}{$\begin{array}{l}\text { 10:30-13:30 } \\
\text { 14:30-18:00 }\end{array}$} & $\begin{array}{l}24 / 11 / 2017 \\
\text { (Full Day) }\end{array}$ & 1014.6 & 0.89 & 0.67 & 262.7 & 0.26 & 0.78 & 0.73 & 792.7 & 0.298 \\
\hline & $\begin{array}{l}\text { 24/11/2017 } \\
\text { (Afternoon) }\end{array}$ & 1007.58 & 0.89 & 0.68 & 212.7 & 0.21 & 0.78 & 0.73 & 252.0 & 0.25 \\
\hline \multirow[t]{2}{*}{$\begin{array}{l}\text { 07:00-11:00 } \\
\text { 12:00-16:00 } \\
\end{array}$} & $\begin{array}{l}25 / 11 / 2017 \\
\text { (Full Day) }\end{array}$ & 2419.1 & 0.95 & 0.93 & 547.6 & 0.23 & 0.82 & 0.96 & 718.0 & 0.297 \\
\hline & $\begin{array}{l}25 / 11 / 2017 \\
\text { (Afternoon) } \\
\end{array}$ & 1890.82 & 0.94 & 1.00 & 460.7 & 0.24 & 0.91 & 1.00 & 473.7 & 0.25 \\
\hline \multirow[t]{2}{*}{$\begin{array}{l}\text { 07:00-11:00 } \\
\text { 12:00-16:00 }\end{array}$} & $\begin{array}{l}\text { 26/11/2017 } \\
\text { (Full Day) }\end{array}$ & 3207.5 & 0.87 & 0.92 & 749.8 & 0.23 & 0.74 & 0.92 & 912.3 & 0.284 \\
\hline & $\begin{array}{l}\text { 26/11/2017 } \\
\text { (Afternoon) }\end{array}$ & 1212.21 & 0.87 & 0.78 & 301.0 & 0.25 & 0.68 & 0.80 & 332.3 & 0.27 \\
\hline
\end{tabular}
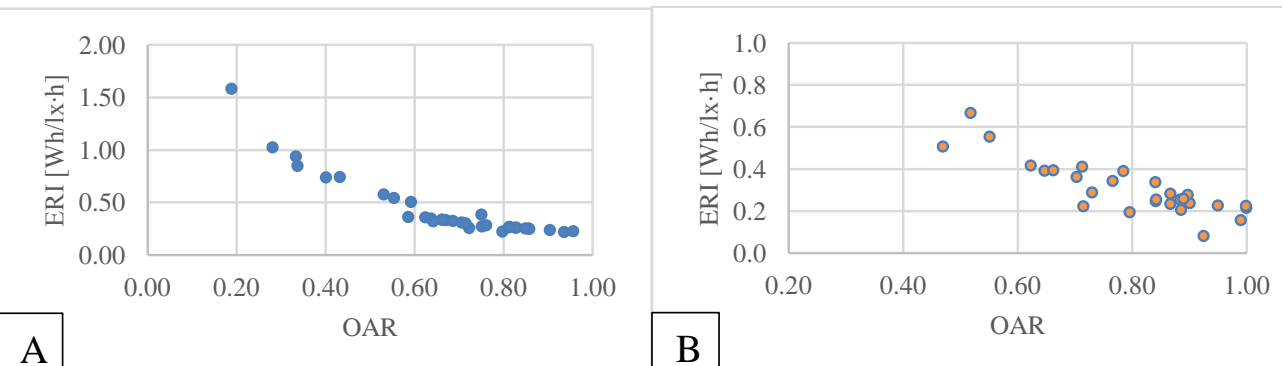

Fig.7: Relation between ERI and OAR calculated for the case study in Lampedusa (A) and for the case study in Palermo (B).

In Figure 7, it is possible to observe that a better relationship between ERI and OAR has been found for the case study A. Nevertheless, as it is possible to appreciate in Table 2, there are more cases when the values of OAR calculated for the case study A are lower than 0.5. Furthermore, in some case of case study B the value 
of OAR was 1. It is worth underlining that both OAR and UAR should be calculated to have a comprehensive outline of DLCs achievement. Indeed, it is possible that the system did not address problems of overilluminance, but gave problems of under-illuminance and vice versa.

In consequence, it is not easy comment on the relation between UAR and ERI because in most cases the values of UAR are equal to 1 because there were no problems of under-illuminance.

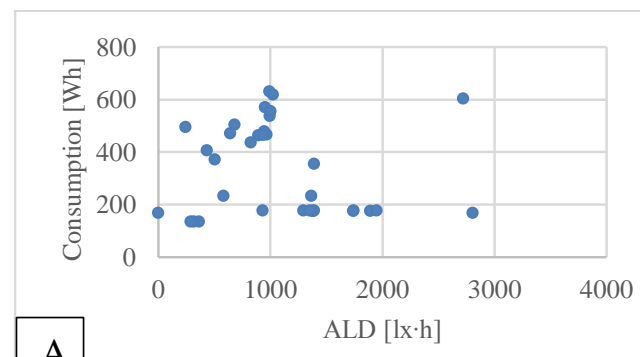

A

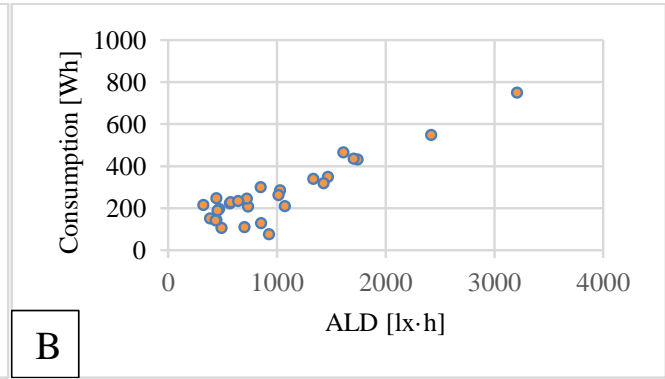

B

Fig. 8: Relation between ALD and consumption calculated for the case study in Lampedusa (A) and for the case study in Palermo (B).

In general, looking at the ALD values, the range of daylight contributes is roughly the same. Nevertheless, looking at figure 9A and 9B, it is possible to see that there is a certain proportionality between ALD and consumption, but only for the case study B. As well for this case, only in an ideal situation, there would be a perfect relation between ALD and consumption and UAR and OAR would be equal to 1. It was observed that high values of ALD correspond to a small change to have glare problems and, therefore, high values of OAR.
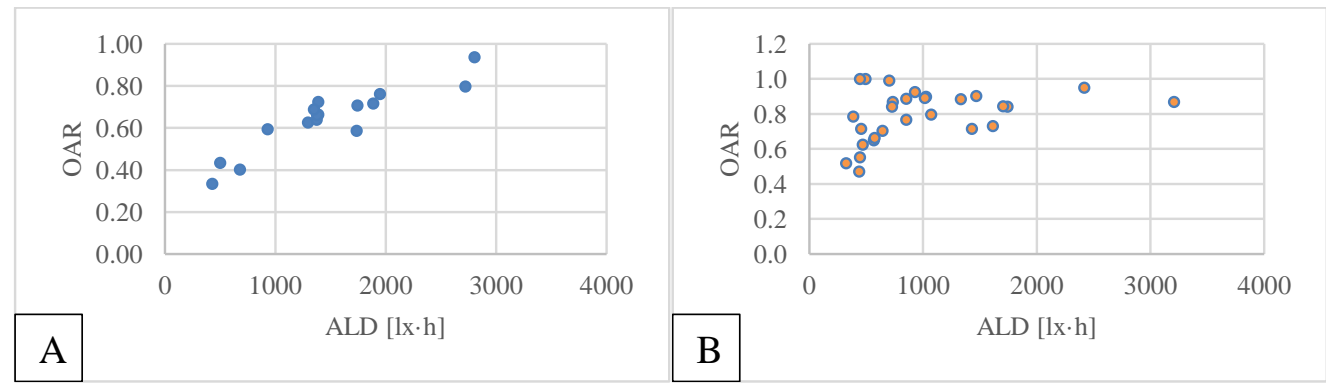

Fig. 9: Relation between ALD and OAR calculated for the case study in Lampedusa (A) and for the case study in Palermo (B).

\section{Conclusion}

Actual performances of the daylight-linked control system were studied using a set of indices presented in the literature by the authors of this work. Rather than highlighting a malfunctioning of the system under observation, results confirmed that: the control systems of the lighting rarely manage to make an ideal adjustment of the luminous flux due to the intrinsic complexity in the relations between the luminous quantities internal and external. Nonetheless, their effectiveness in terms of reducing consumption is never negligible.

These indices want to evaluate the excess and the deficit of illuminance based on a setpoint value on the workplane. It should be achieved and maintained if the control system works ideally. The indices considered the excess or the deficit of lighting respect to a setpoint value of illuminance. They have been calculated for different scenarios characterized by different sky condition and different control strategies. The comparison between the indices calculated for two case studies demonstrated that the same system could work in a different way if installed in spaces characterized by different geometry, latitude and light systems.

From these analyses it has been found that high values of OAR (reduced frequency of lighting excesses) were related to low values of ERI (related to energy efficiency). The calculated values of OAR have shown that the control system has not always worked ideally. On the contrary, the calculation of the UAR indices (an under illuminance flag) testified that, with the exception of very few cases, the system did not give problems of "under-lighting". The relationship between ALD (artificial light demand) and consumption shows a certain proportionality, but not perfect (e.g. with UAR and OAR equal to 1). It also turned out that the ALD values depend on the lower chance of having glare problems and therefore values of OAR value. 
The higher ALD values correspond to the lower possibility of having glare problems and therefore high OAR values. These results, confirm what it has been shown in previous research.

Indeed, in average, the system worked better in Palermo in terms of OAR, both with dimmer and on-off strategy; while worked better in Lampedusa in terms of UAR. Nevertheless, the comparison between the ALD and the actual consumption show that there is a better relation for the indices calculated for the case study in Lampedusa. Being the ALD and indicator useful to understand how the system should work in terms of consumption, this analysis underlined that gap between the ideal performance and the actual ones is bigger in the case study in Palermo. Many reasons can cause these malfunctioning such as the positioning of the photosensors (not adequate for a certain case study) or the calibration procedure. The application of methods to properly design and install the control systems can help to avoid or reduce the cited problems of over-lighting and under-lighting.

\section{Acknowlodgements}

This work is mainly based on the output of the research Ricerca di Sistema Elettrico. Progetto D.1 - Analisi sperimentale e numerica di tecnologie solar driven per la climatizzazione e la produzione di ACS e di sistemi ICT per il controllo e la riduzione dei carichi elettrici nelle isole minori non connesse alla RTN (PAR 2017 CUP I12F17000070001), funded by the Italian Minister of Economic Development and Managed by ENEA in cooperation with Università degli Studi di Palermo, Italy.

\section{References}

[1] Beccali, M., Bonomolo, M., Ciulla, G., \& Brano, V. L. (2018). Assessment of indoor illuminance and study on best photosensors' position for design and commissioning of Daylight Linked Control systems. A new method based on artificial neural networks. Energy, 154, 466-476.

[2] Bellia, L., Fragliasso, F., \& Pedace, A., 2015. Lighting control systems: factors affecting energy savings' evaluation. Energy Procedia, 78, 2645-2650.

[3] Bonomolo, M., Beccali, M., Brano, V. L., \& Zizzo, G., 2017. A set of indices to assess the real performance of daylight-linked control systems. Energy and Buildings, 149, 235-245.

[4] Domingues, P., Carreira, P., Vieira, R., \& Kastner, W. (2016). Building automation systems: Concepts and technology review. Computer Standards \& Interfaces, 45, 1-12.

[5] EN 12464:1 "Light and lighting-Lighting of workplaces. Part 1: Indoor work places"

[6] Jain, S., \& Garg, V. (2018). A review of open loop control strategies for shades, blinds and integrated lighting by use of real-time daylight prediction methods. Building and Environment, 135, 352-364 